\title{
Genetic code as an image of the mirror image. Supplement 1
}

\author{
Miloje M. Rakočević \\ University of Niš, Faculty of Sciences and Mathematics, Department of Chemistry, \\ Višegradska 33, 18000 Niš, Serbia
}

\begin{abstract}
Searching for the answer to the question why - in the generating of the genetic code - only mirror symmetrical left and not right amino acids (AAs) were selected, in a previous work we showed the existence of a double Boolean "triangle" in mirror symmetry, with superposition of the top vertices: 00 -11-22 / 22-11-00 $\rightarrow$ 00-11-22-11-00 [0 as 000; 1 as 001; 2 as 010] (Rakočević, 2019a). The resulting sequence, summed with the binary sequence of a 6-bit binary tree, split with a mirror in the middle (101/010) [as in Dirac's positron / electron mirror], results in a sequence of decimal number system: 02-13-24-16-05, where a smaller number $(010=2)$ was added three times and a larger number $(101=5)$ twice (Survey 1$)$. The mirror image of the obtained decimal sequence (20-31-42-61-50) is 100\% consistent with the arrangement of protein AAs, arranged according to strict chemical similarity (Rakočević, 2019a, Table 3). Starting from this result, the paper of which this is a supplement, presents new insights and new examples of mirror symmetry valid for the genetic code, showing that mirror symmetry is also in other respects an essential feature of the genetic code. In this Supplement are given the further new insights.
\end{abstract}

Keywords: Protein amino acids, Genetic code, Binary tree, Golden mean, Periodic system, Mirror image. Mirroring.

With all that said in the original paper on "Genetic code as an image of the mirror image" (Rakočević, 2019b) ${ }^{1}$ of which this text is a Supplement, here are given some additional new insights into the genetic code (GC). First, we show that it makes sense to test the existence of balancing and nuancing ${ }^{2}$ of the number of atoms in amino acid molecules, correspondent with their (molecular) physicochemical properties in $\mathrm{PAAS}^{3}$ in a new way. First, with the distinction through the first four AAs, which are the first and in their stereochemical types: Gly, the first, i.e. the simplest (and only) in the glycine type;

\footnotetext{
${ }^{1}$ It is understood that the reader is also familiar with the previous article "Protein amino acids as a complete (periodic) system" (Rakočević, 2019a).

${ }^{2}$ The notions of balancing and nuancing related to the state of the genetic code, as well as the conditions of

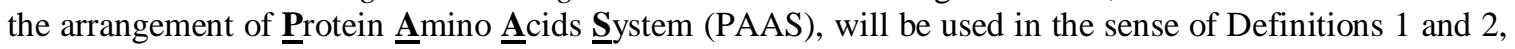
given in our previous paper (Rakočević, 2018a, p. 33).

${ }^{3}$ Rakočević, 2019a: "... the most complete Protein Amino Acids System (PAAS). It is shown that 20 protein amino acids appear to be a complete system - ordered, coherent, and harmonic. In such a system, all chemical distinctions within the system are accompanied by specific arithmetical and algebraic regularities, including the existence of amino acid ordinal numbers from 1 to 20 . The classification of amino acids into two decades (1-10 and 11-20) appears to be in a strict correspondence with the balances of the number of atoms" (Rakočević, 2019a, Table 2, p. 14).
} 
Ala, the first, i.e. the simplest in the set of 16 AAs of alanine stereochemical type; Val, the first, i.e. the simplest in the set of two AAs of valine stereochemical type; Pro, the first, i.e. the simplest (and only) in the proline type. ${ }^{4}$ The result given in Table 1 is proof of the affirmation of this query. The next symmetric and chemically meaningful, and questionable, distinction is the division into $(5+5)$ and $(5+5)$ of AAs. The result given in Table 2 is proof of the affirmation of this query. ${ }^{5}$

[Notice the validity of the principle of self-similarity in the relation between the arrangement given in Table 2 and the arrangement given in Table 5, respectively: GAVPI $36+$ NDSTC $33=69$ versus GREN 36 + VSDQ $33=69$; LKRQE $66+$ MFYWH $69=$ 135 versus THMFLP $(66-1)+$ KWCYAI $(69+1)=135$.]

In this analysis, it is important to recall that PAAS was generated based on the chemical similarity of AAs, with strict adherence to the two most important Mendeleevian principles: the continuity principle and the minimum change principle. When this is the case, then it makes sense, except through the golden mean (Rakočević, 1998, 2011), to test the order of AAs on the binary-code tree of GC (Figure 1) and from the neighborhood - non-neighborhood point of chemical pairs of AAs, starting from the simplest AA, glycine. We first find out the existence of neighborhood pairs, and members within pairs, in the alanine type, and the absence of a neighborhood in the simpler pair of non-alanine AAs $(\mathrm{G}-\mathrm{V})$; all together that are the regularities in the set of the first four pairs (Table 3). Also there is the absence of a neighborhood at the next alanine AAs and the neighborhood at a more complex pair of non-alanine AAs (P-I); all together that are the regularities in the set of the next six pairs of AAs (Table 4).

The test results, analogous to the results in Tables 1 and 2, are given in Tables 5 and 6 , in correspondence with Figures $2 \& 3$. Balancing and nuancing of the number of atoms in the side chains of AAs, correspondent to the physicochemical properties of amino acid molecules, are more than obvious.

\section{Box 1. Explanation of Figure 2}

"The Cyclic Invariant Periodic System (CIPS) of canonical AAs. At the [index] - the atom number within amino acid side chains. In the middle position there are chalcogene AAs (S, T \& $C, M$ ); follow - in next 'cycle' - the AAs of non-alanine stereochemical types (G, P \& V, I) [in red color], then two double acidic AAs with two their amide derivatives (D, E \& N, Q) [in blue color], the two original aliphatic AAs with two amine derivatives (A, L \& K, R) [in orange and yellow color]; and, finely, four aromatic AAs $(\mathrm{F}, \mathrm{Y} \& \mathrm{H}, \mathrm{W})$ - two up and two down. ... Notice that each amino acid position in this CIPS is strictly determined and none can be changed" (Rakočević, 2011, Fig. 6, p. 632). Note in this Supplement: In the previous paper (Rakočević, 2019a, Box 4) the CIPS (as here in Figure 2) was called CIPS I, because we introduced CIPS II there, and now we are introducing CIPS III in Figure 3.

\footnotetext{
${ }^{4}$ On the four stereochemical types of AAs see in (Popov, 1989; Rakočević and Jokić, 1996).

5 It should be noted that the quantities 97-107 in Table 1 are directly related to sequence 25-36-61, the determinant of both chemical and genetic code (Rakočević, 2019a, Table A2, p. 29).
} 
Unlike the cyclicity in CIPS I, where cyclicity is expressed through pairs of AAs, in CIPS III cyclicity is more complex. First, we note the simultaneous similarity and difference with CIPS I. Namely, in CIPS I, the outer cycle consists of aromatic AAs, unlike all other aliphatic ones. On the other hand, in CIPS III, the outer cycle consists of AAs of the non-alanine stereochemical type, unlike any other belong to the alanine stereochemical type. The interior of CIPS I consists of AAs, as described in the legend of Figure 2 (given here in Box 1). The interior of CIPS III (in Figure 3) is chemically more complex in the following sense: in the upper part, in the outer "ring" of four AAs with simpler nitrogen-oxygen functions, four AAs, with more complex nitrogen-oxygen functions are closed; in the lower part, however, there are four aromatic AAs in the outer "ring" and in the interior there are only two sulfur AAs, aliphatic but different from all other aliphatic [in their molecules except the same four atoms $(\mathrm{H}, \mathrm{C}, \mathrm{N}, \mathrm{O})$ they also have a fifth $(\mathrm{S})$ as a "hetero" atom].

\section{References}

Popov, E.M., 1989. Strukturnaya organizaciya belkov. Nauka, Moskva.

Rakočević, M.M., 1998a. The genetic code as a Golden mean determined system. Biosystems, 46, 283-291.

Rakočević, M.M., 2018a. The Cipher of the Genetic Code. BioSystems 171 (2018) 31-47

Rakočević, M. M., 2018b. Analogies of Genetic and Chemical Code. Polyhedron, 153, 292-298.

Rakočević, M. M., 2019a. Protein amino acids as a complete (periodic) system. Chemia Naissensis, Vol 2, Issue 1, pp. 1-43.

Rakočević, M. M., 2019b. Genetic code as an image of the mirror image. Part I, OSF Preprints, DOI 10.31219/osf.io/xht4c.

Rakočević, M.M., Jokić, A., 1996. Four stereochemical types of protein amino acids: synchronic determination with chemical characteristics, atom and nucleon number. J. Theor. Biol. 183, 345-349. 


\section{S u r ve y}

Survey 1. The mirroring of (double) first Boolean triangle and crossing with "Golden sequence" 0-1-0-1-0-1 from the six-bit binary tree ${ }^{6}$

$00-11-22 / 22-11-00 \rightarrow 00-11-22-11-00[0$ as $000 ; 1$ as $001 ; 2$ as 010$]$
$(00-11-22-11-00)+[3(010=2)]+[2(101=5)]=02-13-24-16-05$
$02-13-24-16-05 / 20-31-42-61-50$

\section{F i g u r e s}

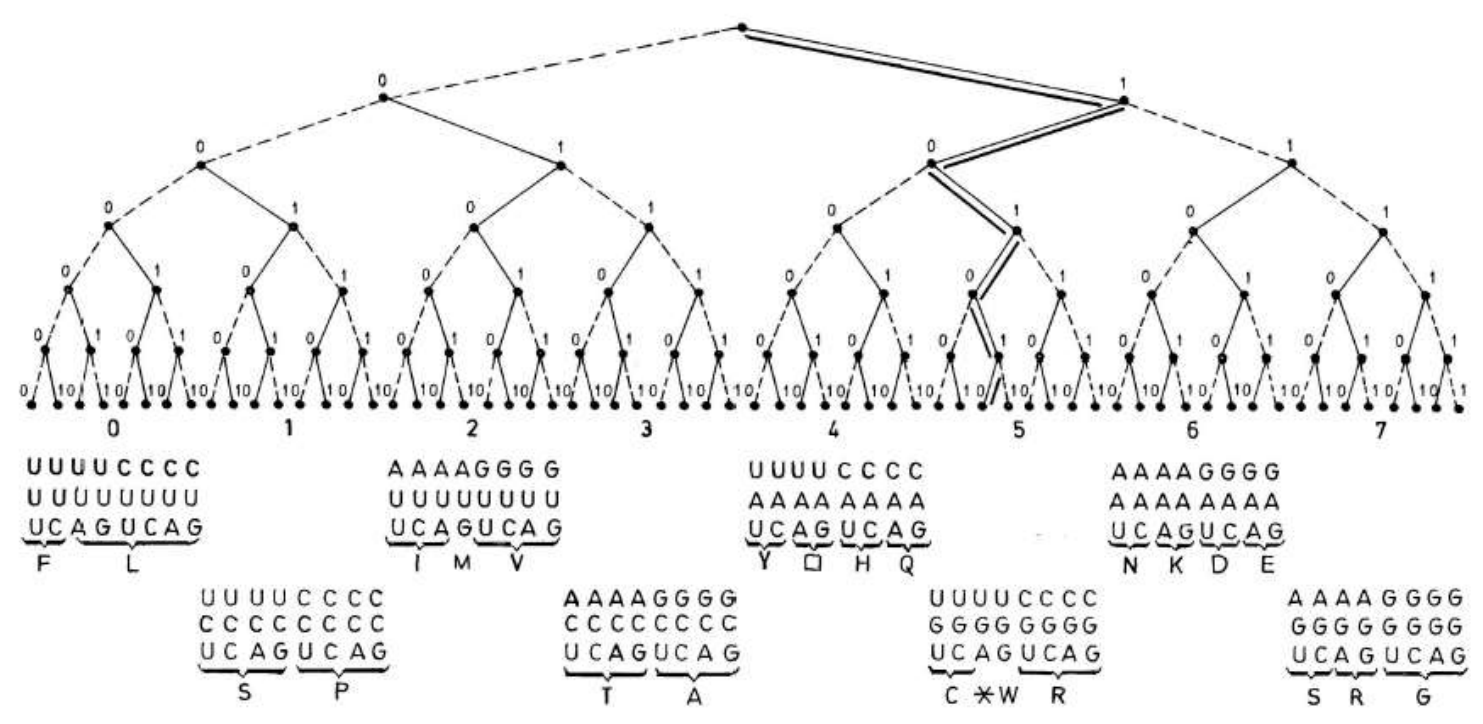

Figure 1. "Genetic code as a binary-code tree. The full lines: the routes of the greater (faster) changes from pyrimidine to purine or from two to three hydrogen bonds and vice versa. The dotted lines: the routes of the less (slower) changes. The double full line: the route of the maximum possible (fastest) changes; the route corresponding to the 'Golden mean route' on the Farey tree ... " (Rakočević, 1998, Fig. 1, p. 284).

\footnotetext{
6 The sequence 0-1-0-1-0-1 from the six-bit binary tree is a "Golden sequence" through the correspondence between the binary tree and Farey tree as we explained in a previous paper (Rakočević, 1998, Fig. 1 in relation to Fig. 2).
} 


\begin{tabular}{|cc|cc|}
\hline $\mathrm{F}_{14}$ & $\mathrm{Y}_{15}$ & $\mathrm{G}_{01}$ & $\mathrm{~V}_{10}$ \\
$\mathrm{~L}_{13}$ & $\underline{\mathrm{A}}_{04}$ & $\mathrm{R}_{17}$ & $\mathrm{~S}_{05}$ \\
$\mathrm{Q}_{11}$ & $\mathrm{~N}_{08}$ & $\mathrm{E}_{10}$ & $\mathrm{D}_{07}$ \\
$\mathrm{P}_{08}$ & $\mathrm{I}_{13}$ & $\mathrm{~N}_{08}$ & $\mathrm{Q}_{11}$ \\
$\mathrm{~T}_{08}$ & $\mathrm{M}_{11}$ & $\mathrm{~K}_{15}$ & $\mathrm{~T}_{08}$ \\
$\mathrm{~S}_{05}$ & $\mathrm{C}_{05}$ & $\mathrm{~W}_{18}$ & $\mathrm{H}_{11}$ \\
$\mathrm{G}_{01}$ & $\mathrm{~V}_{10}$ & $\mathrm{C}_{05}$ & $\mathrm{M}_{11}$ \\
$\mathrm{D}_{07}$ & $\mathrm{E}_{10}$ & $\mathrm{Y}_{15}$ & $\mathrm{~F}_{14}$ \\
$\mathrm{~K}_{15}$ & $\mathrm{R}_{17}$ & $\mathrm{~A}_{04}$ & $\mathrm{~L}_{13}$ \\
$\mathrm{H}_{11}$ & $\mathrm{~W}_{18}$ & $\mathrm{I}_{13}$ & $\mathrm{P}_{08}$ \\
Figure 2. CIPS I (Box 1) & Figure 3. CIPS III \\
\hline
\end{tabular}

\section{T a b l e s}

Table 1. The first four and following AAs in PAAS (Rakočević, 2019a)

\begin{tabular}{|c|c|c|c|c|}
\hline $\mathrm{G}_{01}$ & $\mathrm{~N}_{08}$ & & & \\
\hline $\mathrm{A}_{04}$ & $D_{07}$ & 23 & 28 & $\rightarrow 51$ \\
\hline$V_{10}$ & $\mathrm{~S}_{05}$ & & & $(1 \times 51)$ \\
\hline $\mathrm{P}_{08}$ & $\mathrm{~T}_{08}$ & & Cross & $\rightarrow 107 / 97$ \\
\hline $\mathrm{I}_{13}$ & $\mathrm{C}_{05}$ & & & \\
\hline $\mathrm{L}_{13}$ & $M_{11}$ & & & \\
\hline $\mathrm{K}_{15}$ & $F_{14}$ & & & \\
\hline $\mathrm{R}_{17}$ & $Y_{15}$ & 79 & 74 & $\rightarrow 153$ \\
\hline $\mathrm{Q}_{11}$ & $W_{18}$ & $\downarrow$ & $\downarrow$ & $(3 \times 51)$ \\
\hline$E_{10}$ & $\mathrm{H}_{11}$ & 102 & 102 & \\
\hline
\end{tabular}


Table 2. The first five and following AAs in PAAS (Rakočević, 2019a)

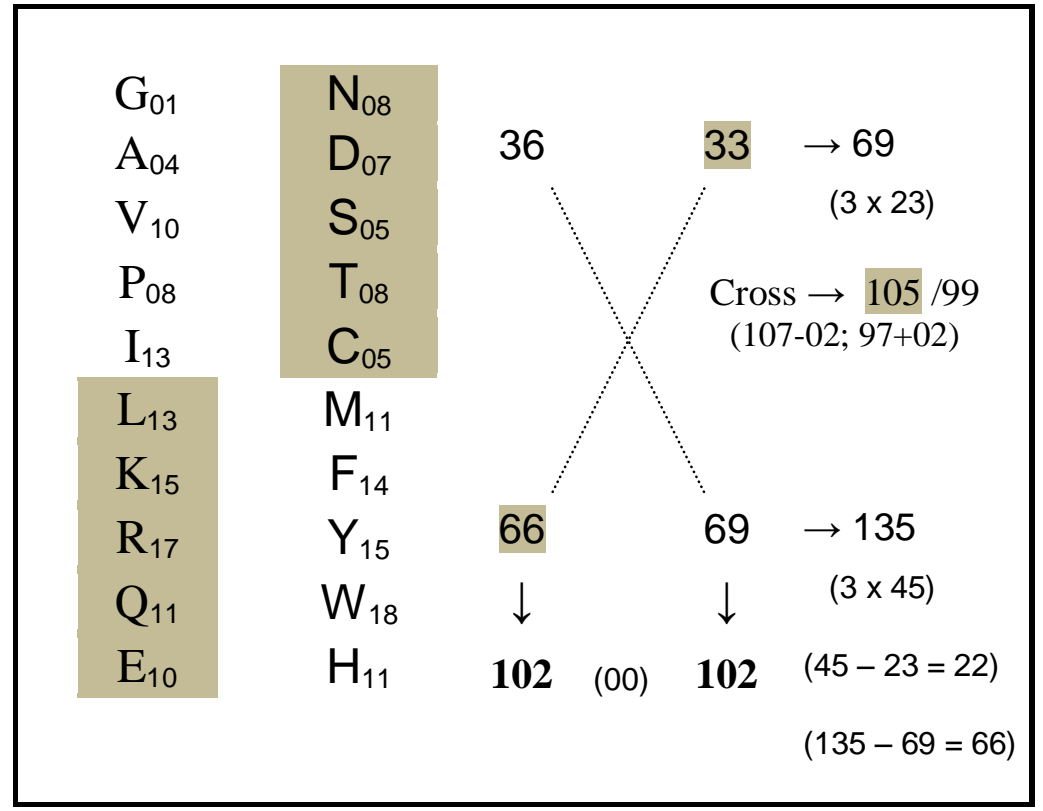

Table 3. Mostly adjacent AAs

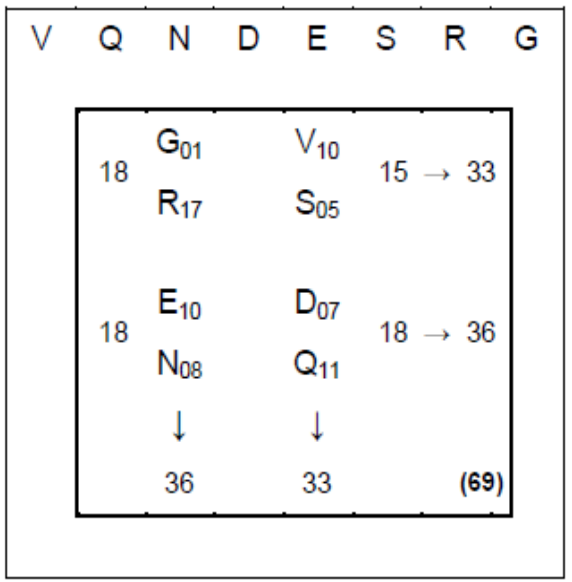

The adjacencies on Binary tree (Fig. 1)
Table 4. Mostly non-adjacent AAs

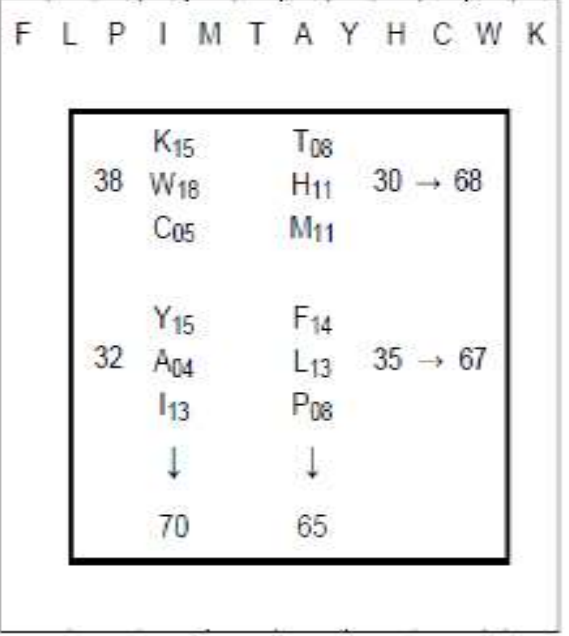


Table 5. The first four and following six AAs pairs

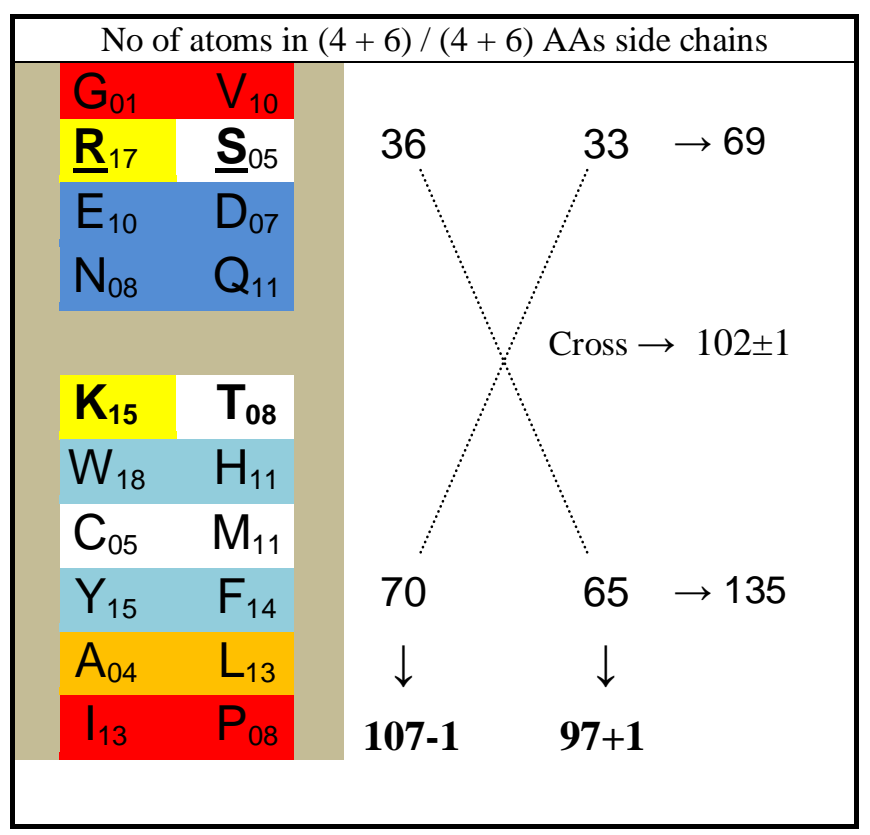

Table 6. The first five and following five AAs pairs

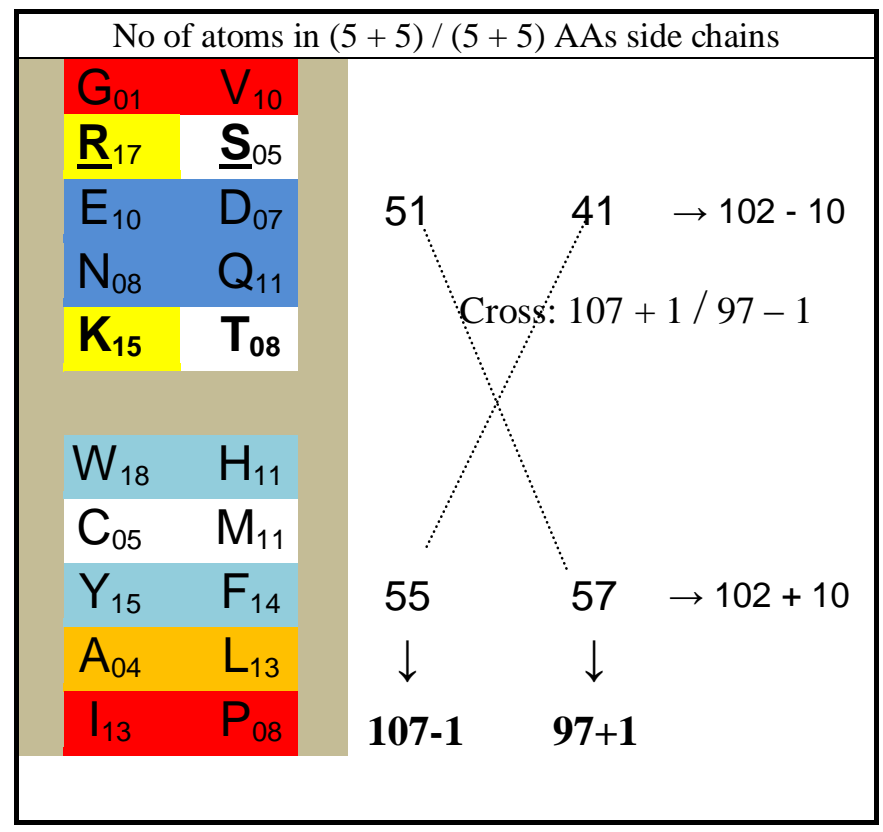

